\title{
Chiral discrimination between tyrosine and $\beta$-cyclodextrin revealed by cryogenic ion trap infrared spectroscopy
}

\author{
Keisuke Hirata, ${ }^{1,2}$ Yuta Mori, ${ }^{1,2}$ Shun-ichi Ishiuchi* ${ }^{1,2}$ Masaaki Fujii*1,2,3 and Anne Zehnacker*3,4 \\ ${ }^{1}$ Laboratory for Chemistry and Life Science, Institute of Innovative Research, Tokyo Institute of \\ Technology, 4259, Nagatsuta-cho, Midori-ku, Yokohama, 226-8503, Japan. \\ ${ }^{2}$ School of Life Science and Technology, Tokyo Institute of Technology. 4259 Nagatsuta-cho, Midori-ku, \\ Yokohama, Kanagawa, 226-8503, Japan. \\ ${ }^{3}$ Tokyo Tech World Research Hub Initiative (WRHI), Institute of Innovation Research, Tokyo Institute of \\ Technology, 4259, Nagatsuta-cho, Midori-ku, Yokohama, Japan. \\ ${ }^{4}$ Université Paris-Saclay, CNRS, Institut des Sciences Moléculaires d'Orsay, 91405, Orsay, France.
}

\section{Abstract}

Complexes of permethylated $\beta$-cyclodextrin ( $\beta-M C D)$ with the two enantiomers of protonated tyrosine (L- and $\mathrm{D}-\mathrm{TyrH}^{+}$) are studied by cryogenic ion trap infrared photo-dissociation spectroscopy. The vibrational spectra in the $\mathrm{OH} / \mathrm{NH}$ stretch and fingerprint regions are assigned based on density functional theory calculations. The spectrum of both $\mathrm{L}$ - and $\mathrm{D}-\mathrm{TyrH}^{+}$complexes contains features characteristic of a first structure with ammonium and acid groups of the amino acid simultaneously interacting with the $\beta-\mathrm{MCD}$, the phenolic $\mathrm{OH}$ remaining free. A second structure involving additional interaction between the phenolic $\mathrm{OH}$ and the $\beta-\mathrm{MCD}$ is observed only for the complex with $\mathrm{D}-\mathrm{TyrH}^{+}$. The larger abundance of the $\mathrm{D}-\mathrm{TyrH}^{+}$complex in the mass spectrum is tentatively explained in terms of (1) better insertion of D-TyrH ${ }^{+}$within the cavity with the hydrophobic aromatic moiety less exposed to hydrophilic solvent molecules and (2) a stiff structure involving three interaction points, namely the ammonium, the phenolic $\mathrm{OH}$ and the carboxylic acid $\mathrm{OH}$, which is not possible for the complex with L$\mathrm{TyrH}^{+}$. The recognition process does not occur through size effects that induce complementarity to the host molecule but specific interactions. These results provide a comprehensive understanding of how the cyclodextrin recognises a chiral biomolecule. 


\section{Introduction}

Cyclodextrins (CDs) are cavity-forming macrocyclic oligosaccharides. Due to their toroidal shape with a hydrophobic interior and a hydrophilic exterior, they are widely used for their capacity to form inclusion complexes with hydrophobic molecules, enhancing thereby their water solubility. These unique properties make them widely used as vectorisation agents of drugs, ${ }^{1,2}$ or stationary phases in chromatography, ${ }^{3}$ for the separation of structural isomers or enantiomers. ${ }^{4}$ Indeed, many chiral stationary phases (CSPs) are based on cyclodextrin, like the Astec CYCLOBOND ${ }^{\mathrm{TM}}$.

However, the mechanism by which these chromatography columns separate enantiomers is still not fully understood. A major question arises as to the large gap between the cavity size of $C D$ and the size of general guest molecules. For instance, $\beta-C D$, made of seven glycose units linked by $\alpha-1,4$ glycosidic bonds, is adapted to a wide range of small guest molecules such as amino acids. Nevertheless the cavity size of $\beta-C D$ is much larger than that of amino acids. A general "three-point interaction" model proposes that chiral discrimination requires at least three interactions between host $C D$ and guest molecules, one at least being enantiospecific. ${ }^{4-6}$ Interestingly, this model suggests that chiral discrimination is largely achieved by specific interactions rather than by size matching between $C D^{\prime} s$ cavity and guest molecules although structural complementarity is generally thought to be a major reason for substrate specificity. ${ }^{7}$ The enantiomer with higher affinity to $C D$ is assumed to be deeply included in the hydrophobic cavity of CD (inclusion mode) rather than to be located in the vicinity of CD's outer rim surface (surface binding mode) just because the three-point interaction model demands multiple (more than two) interaction points. ${ }^{4}$

Mass spectrometry methods based on the exchange rate between the amino-acid and a non-chiral reactant have been proposed as a powerful tool for determining enantiomeric excess. ${ }^{85,9}$ In these experiments, the enantioselectivity of the interaction is defined by the $k_{L} / k_{D}$ ratio between the displacement rate constants. ${ }^{10}$ This method has been applied to the $\beta-C D /$ amino acid systems. Based on molecular modelling, these experimental findings were tentatively explained in terms of a binding pattern involving whole inclusion of the amino acids, with both the amino and carboxylic acid parts interacting with the primary ring. ${ }^{5}$ Although mass spectrometry allows studying the host-guest interaction without the perturbation of the solvent, it is difficult to rationalise the observed behaviour in terms of structure. The observed selectivity rests on kinetic measurements, and both energy difference between the diastereomeric host-guest complexes and barrier heights may play a role. In this context, vibrational spectroscopy is very useful to get direct structural information on host-guest complexes and reveal the relationship between chiral discrimination by $C D$ and the structure of the complexes. Several studies combining mass spectrometry and laser spectroscopy have been conducted so far to understand chirality recognition in the gas phase ${ }^{11-14}$, in particular in the field of 
chiral supramolecular chemistry. ${ }^{15-17}$ Combination of ion mobility spectrometry (IMS) and infrared multiple-photon dissociation (IRMPD) spectroscopy has been used to define the difference in binding patterns between $L$ and $D$ alanine or $L$ and $D$ isoleucine in permethylated $\beta-C D(\beta-M C D) .{ }^{18,} 19$ Interestingly, very different vibrational spectra were obtained for the two enantiomers of isoleucine, which were assigned to higher-energy species resulting from kinetic trapping. However, due to the lack of mass spectrometry data on which enantiomer is preferentially complexed with $\beta-M C D$, and to what extent, the mechanism of chiral discrimination has not been correlated to molecular structure.

We report here the spectroscopic study of the complex of $\beta$-MCD with the two enantiomers of protonated tyrosine (denoted as L- and D-TyrH ${ }^{+}$) using cryogenic ion trap technique. ${ }^{20}$ In contrast to the previously studied amino-acids, protonated tyrosine possesses a phenol aromatic ring, which provides an additional hydrogen bonding site. The low temperature achieved allows better spectral resolution than room-temperature experiments. Recently, the combination of cryogenic ion traps with conformer-specific spectroscopy has been used to study chiral recognition in protonated glutamic acid dimers ${ }^{21}$ or systems mimicking the interaction between a neurotransmitter and its receptor. ${ }^{22}$ This work aims at defining the binding mode for the L-TyrH ${ }^{+}$and D-TyrH ${ }^{+}$-containing host-guest complex and understand whether it is influenced by the chirality of the guest or not. Several sites of $\mathrm{TyrH}^{+}$, i.e. the ammonium $\mathrm{NH}$, the phenolic $\mathrm{OH}$, and the carboxylic $\mathrm{OH}$, can indeed interact with $\beta-\mathrm{MCD}$ and we discuss the role of these interactions in the chirality recognition process. The molecules under study are shown in Figure 1.

\section{Experimental and theoretical methods}

\section{1) Experimental methods}

The experimental protocols we employed are essentially the same as described in previous articles. ${ }^{23}$ ${ }^{24}$ Briefly, the methanol solutions containing $\beta-\mathrm{MCD}\left(5-7 \times 10^{-5} \mathrm{M}\right)$ and L- or D-Tyr $\left(0.7-2 \times 10^{-5} \mathrm{M}\right)$ with $0.5 \%$ of formic acid were electrosprayed from a nebuliser to generate fine droplets containing the target complexes. The droplets were dried up by travelling through a glass capillary heated up to 60 ${ }^{\circ} \mathrm{C}$. The ions efficiently collected by an ion funnel were injected into a quadrupole mass spectrometer (Q-MS: Extrel) via a hexapole ion guide. The target host-guest complexes were mass-selected by the Q-MS and then introduced to a cryogenic quadrupole ion trap (QIT) via a quadrupole ion bender and octupole ion guides. The QIT was kept at $4 \mathrm{~K}$ by a closed-cycle two-stage He refrigerator (Sumitomo: RDK-408D2) during the whole experiments. A mixed buffer gas of $\mathrm{H}_{2}(20 \%)$ and helium was introduced to the QIT using a pulsed valve (Parker Hannifin: General Valve Series 9). The mass-selected ions were allowed to collide with the cold buffer gas so that they were trapped in the QIT and cooled down to 
$\sim 10 \mathrm{~K}$. Hydrogen molecules were allowed to weakly attach to the ions under such cryogenic conditions. The $\mathrm{H}_{2}$-tagged ions were irradiated with a tunable IR laser (LaserVision: OPO/OPA). Hydrogen molecules were detached from the cluster ions when the wavenumber of the IR laser is resonant to a vibrational transition of the cluster ions. ${ }^{25,} 26$ The photofragments were detected by a time-of-flight mass spectrometer (TOFMS). The photofragment ion signals from a dynode converter detector of TOFMS were amplified 10 times by a pre-amplifier and recorded on a fast digitiser. IR photodissociation (IRPD) spectra were measured by monitoring the photofragments $(\mathrm{m} / \mathrm{z} 1611)$ intensity as a function of the wavenumber of the IR laser.

2) Theoretical methods and nomenclature of the complexes

The structures of the bare protonated tyrosine $\left(\mathrm{TyrH}^{+}\right)$and its complex with $\beta-\mathrm{MCD}$ were optimised in the frame of the density functional theory (DFT) using the cam-B3LYP functional and the 6-31G(d,p) basis sets. This level of calculation was justified by the size of the system and its validity checked on the example of L-TyrH (vide infra). All the calculations were performed with the Gaussian16 B01 software. ${ }^{27}$ The frequencies were calculated at the same level of theory and scaled by $0.961 .{ }^{28}$ Although no experimental determination of the gas-phase proton affinity of $\beta-M C D$ is available, there is evidence of much higher proton affinity of Tyr than CDs $\left(926 \mathrm{~kJ} / \mathrm{mol}\right.$ vs. less than $800 \mathrm{~kJ} / \mathrm{mol}$, respectively). ${ }^{9}$ For this reason, only the complexes of neutral $\beta-\mathrm{MCD}$ and protonated Tyr were taken in to account (vide infra). ${ }^{9,29}$ We performed optimisation of structures involving either $\mathrm{NH} . . . \mathrm{O}$, phenolic $\mathrm{OH} . . . \mathrm{O}$, carboxylic $\mathrm{OH}$...O interactions, with the three different oxygen atoms, i.e. primary or secondary rim oxygen as well as $\alpha-1,4$ glycosydic linkage oxygens. The starting points were obtained from the potential energy surface (PES) exploration using the Monte Carlo Multiple Minimum method implemented in the MacroModel program ${ }^{30}$ associated to the MMFFs force field. ${ }^{31}$ Exploration were performed both in vacuum and in a methanol solvation continuum. ${ }^{32}$ To ensure systematic exploration of the PES, the structures found for the $\beta$-MCD complex of each enantiomer were systematically modified to provide new starting structures for the other system.

In what follows, we denote phenolic and carboxylic $\mathrm{OHs}$ as $\mathrm{OH}_{\text {phenol }}$ and $\mathrm{OH}_{\text {acid }}$, respectively. $\mathrm{O}_{\text {prim, }} \mathrm{O}_{\text {sec }}$, and $\mathrm{O}_{\alpha}$ indicate oxygen atoms of the primary rim, secondary rim, and $\alpha-1,4$ glycosydic linkage, respectively. We shall use the following notation for each conformer: free and bound $X H(X=N, O)$ groups are denoted by $\mathbf{f}$ and $\mathbf{b}$, respectively. Then, the notation is given in the order of phenolic $\mathrm{OH}$, acid $\mathrm{OH}$, and $\mathrm{NH}_{3}$ groups. If two $\mathrm{NH}$ bonds are hydrogen bonded, we denote it as $\mathbf{b}_{\mathbf{d}}$. For example, a complex of D-TyrH ${ }^{+}$and $\beta-\mathrm{MCD}$ with a bound phenolic $\mathrm{OH}$, a free acid $\mathrm{OH}$, and two bound $\mathrm{NHs}$ is denoted as D-TyrH ${ }^{+}-\mathbf{b f b}_{\mathbf{d}}$. 


\section{Results and Discussion}

1) Mass spectrometry

Figure 2 shows the electrospray ionisation (ESI) TOF mass spectrum of a mixture of $\beta$-MCD with an equivalent amount of D-Tyr (M. W. = 181) and isotope-labelled $\left({ }^{15} \mathrm{~N},{ }^{13} \mathrm{C}\right) \mathrm{L}-\mathrm{Tyr}(\mathrm{M} . \mathrm{W} .=191)$. The peaks $A$ and $B$ at $m / z 1612$ and 1622 correspond to the D-TyrH $-\beta-M C D$ and L-TyrH ${ }^{+}-\beta-M C D$ complexes, respectively. Peak $A$ is more intense than peak $B$, which is more clearly indicated by a high-resolution MS spectrum by Q-MS (Figure 2 inset). This indicates that D-Tyr complex is more abundant than L-Tyr complex.

2) Experimental vibrational spectroscopy

a) Isolated $\mathrm{TyrH}^{+}$

Figure 3a shows a vibrational spectrum of L-TyrH ${ }^{+}$, obtained by IR-UV double resonance spectroscopy, by probing the band located at $35082 \mathrm{~cm}^{-1}$. In the $3 \mu \mathrm{m}$ region, the spectrum is identical to that previously reported for the conformer $\mathrm{A}$ of $\mathrm{TyrH}^{+}{ }^{23,33}$ The free phenolic and carboxylic $\mathrm{OH}$ stretching modes $(\mathrm{v}(\mathrm{OH}))$ are independent of the conformers and appear at 3640 and $3550 \mathrm{~cm}^{-1}$, while the free and bound $v(\mathrm{NH})$ show more versatility and appear in the $3320-3370 \mathrm{~cm}^{-1}$ and $3040-3160 \mathrm{~cm}^{-1}$ ranges, depending on the conformers (Figure S1). In the Amide I and II region, the strong peak at $1780 \mathrm{~cm}^{-1}$ is the signature of the $v(\mathrm{CO})$ of the acid group (Figure 4a), which confirms the finding in the $3 \mu \mathrm{m}$ range that $\mathrm{TyrH}^{+}$is not in a zwitterionic form. In the fingerprint region, the spectrum is similar to the IRMPD spectrum at room temperature. ${ }^{34}$ We assigned the bands below $1700 \mathrm{~cm}^{-1}$ on the basis of our theoretical calculations: aromatic $\mathrm{CH}$ bending mode $\left(\beta\left(\mathrm{C}_{\text {arom }} \mathrm{H}\right)\right)$ coupled to aromatic $\mathrm{CC}$ stretch $\left(v\left(\mathrm{CC}_{\text {arom }}\right)\right)$ at 1617 and $1288 \mathrm{~cm}^{-1}, \beta(\mathrm{CH})$ at $1518 \mathrm{~cm}^{-1}, \beta(\mathrm{NH})_{\text {sym }}$ bend at $1435 \mathrm{~cm}^{-1}$, coupled $\beta(\mathrm{NH})_{\text {sym }}$ bend and $v\left(\mathrm{C}-\mathrm{OH}_{\text {acid }}\right)$ stretch at $1407 \mathrm{~cm}^{-1}, \beta\left(\mathrm{OH}_{\text {acid }}\right)$ bend at $1170 \mathrm{~cm}^{-1}$, and $\beta\left(\mathrm{OH}_{\text {phenol }}\right)$ bend at 1156 $\mathrm{cm}^{-1}$. The comparison between the experimental and theoretical spectra is given in Figure S1 and Table S1 of the supplementary information.

a) Isolated $\beta-\mathrm{MCDH}^{+}$

Figure $3 \mathrm{~b}$ shows the IRPD spectra of $\mathrm{H}_{2}$-tagged protonated $\beta$-MCD. It only shows bands due to $\mathrm{CH}$ stretches in the $3 \mu \mathrm{m}$ region. The calculated spectrum of $\beta-\mathrm{MCDH}^{+}$is shown in Figure $\mathrm{S} 2$ of the supplementary information for the sake of comparison and shows that $\beta-\mathrm{MCDH}^{+}$is protonated on the secondary rim oxygen in our experimental condition, with $\mathrm{OH}$ stretch out of our experimental range $\left(\sim 2044 \mathrm{~cm}^{-1}\right)$. 
b) $\beta$-MCD complexes with $\mathrm{TyrH}^{+}$

IR-UV double resonance spectroscopy is not possible for the complexes of $\beta$-MCD studied here because they are stable upon UV irradiation due to the cage effect of $\beta-M C D$, already observed in other systems. ${ }^{35-37}$ The reported spectra are obtained by IRPD of the $\mathrm{H}_{2}$-tagged complexes. Figures $3 \mathrm{c}$ and $\mathrm{d}$ show the vibrational spectra of the $L-\mathrm{TyrH}^{+}-\beta-\mathrm{MCD}$ and D-TyrH ${ }^{+}-\beta-\mathrm{MCD}$ complexes, respectively. In Figure 3, Blue-, purple-, and green-coloured areas correspond to the bands assigned to $v\left(\mathrm{OH}_{\text {phenol }}\right)$, $v\left(\mathrm{OH}_{\text {acid }}\right)$, and $v(\mathrm{NH})$, respectively. The intense bands observed in the $2800-3020 \mathrm{~cm}^{-1}$ range were assigned to the $v(C H)$ stretches of $\beta-M C D$ by comparison with the IRPD spectra of protonated $\beta-M C D$ (Figure 3b). Though no clear difference was observed in the $2800-3020 \mathrm{~cm}^{-1}$ range, the two spectra clearly differ from each other in the region above $3020 \mathrm{~cm}^{-1}$. They both present a sharp transition characteristic of the free $v\left(\mathrm{OH}_{\text {phenol }}\right)$, with however different relative intensity. For both complexes, no free $v\left(\mathrm{OH}_{\text {acid }}\right)$ is observed, suggesting that the $\mathrm{COOH}$ group of Tyr is either deprotonated or forming a strong hydrogen bond with $\beta-\mathrm{MCD}$. The free and bound $v(\mathrm{NH})$ ranges differ in shape for the two enantiomers. The most important difference between them is the transition at $\sim 3430 \mathrm{~cm}^{-1}$ in the D$\mathrm{TyrH}^{+}-\beta-\mathrm{MCD}$ spectrum, which is absent for $\mathrm{L}-\mathrm{TyrH}^{+}-\beta-\mathrm{MCD}$. This transition is reminiscent of a bound $v(\mathrm{OH})$ of the phenol-18-crown-6 ether complex, with the same $v\left(\mathrm{OH}_{\text {phenol }}\right)$ frequency and the same Franck-Condon-like progression resulting from anharmonic coupling with a low frequency mode. ${ }^{38}$ In the fingerprint region (Figure $4 b, c$ ), the four strong transitions between 1400 and $1800 \mathrm{~cm}^{-1}$ are the counterparts of those assigned to the $v(\mathrm{CO})$, the $\beta\left(\mathrm{C}_{\text {arom }} \mathrm{H}\right)$ or the coupled $v\left(\mathrm{CC}_{\text {arom }}\right) / \beta\left(\mathrm{C}_{\text {arom }} \mathrm{H}\right)$ modes, and the superposition of $\beta(\mathrm{NH})_{\text {sym }}$ and $\beta\left(\mathrm{C}-\mathrm{OH}_{\text {acid }}\right)$ transitions in bare $\mathrm{L}-\mathrm{TyrH}^{+}$. Apart from a stronger intensity of the $\beta(\mathrm{NH})_{\text {sym }} / \beta\left(\mathrm{O}-\mathrm{H}_{\text {acid }}\right)$ in the $\mathrm{D}-\mathrm{TyrH}^{+}-\beta-\mathrm{MCD}$ complex, the spectra are similar for the two enantiomers. Interestingly, the $\mathrm{v}(\mathrm{CO})$ frequency is characteristic of the $\mathrm{COOH}$ form, which allows ruling out the presence of a zwitterion in the complex. Indeed, the zwitterion, observed for example in arginine-containing peptides, would appear below $1700 \mathrm{~cm}^{-1} .{ }^{39}$ Moreover, the bound character of the $\mathrm{COOH}$ manifests itself in the $30-40 \mathrm{~cm}^{-1}$ red shift of $v(\mathrm{CO})$ relative to bare $\mathrm{TyrH}^{+}$. A broad absorption between 2700 and $2800 \mathrm{~cm}^{-1}$ in the spectrum of L- or D-TyrH $-\beta-M C D$, which is not observed in $\mathrm{COOH}$ free tyramineH ${ }^{+}-\beta-M C D$ (Figure S3), can be assigned to strongly bound $v\left(\mathrm{OH}_{\text {acid }}\right)$.

The $1450 \mathrm{~cm}^{-1}$ feature, assigned to the superposition of the $\beta(\mathrm{NH})_{\text {sym }} / v\left(\mathrm{C}-\mathrm{OH}_{\text {acid }}\right)$ transitions, is more intense in the L-Tyr than in the D-TyrH ${ }^{+}-\beta-M C D$ complex. This suggests that complexes involving interaction between the ammonium and $\beta-M C D$ are dominant in the L-TyrH ${ }^{+}-\beta-M C D$ complex. Indeed, comparison between experimental and calculated spectra of protonated phenylalanine shows that this band is more intense when $\mathrm{NH}_{3}{ }^{+}$is involved in a hydrogen bond. ${ }^{40}$

We can therefore draw the following conclusions from the experimental findings: First, the spectroscopic signature in the $v(\mathrm{CO})$ stretch region indicates that the proton remains on the guest, and 
that no zwitterion is formed. ${ }^{39}$ This result is in line with isotopic exchange experiments on protonated $C D$, where the 10 times faster proton exchange in free $C D$ than in ammonium-containing $C D$ indicates the formation of an inclusion complex with the proton on the guest. ${ }^{29}$ Moreover, the carboxylic acid $\mathrm{OH}$ group is always $\mathrm{H}$-bonded to the $\beta$-MCD. Second, amino-bound structures with free phenolic $\mathrm{OH}$ are the only ones observed for L-TyrH ${ }^{+}-\beta-M C D$. In contrast, both amino-bound and phenol-bound structures coexist in D-TyrH $-\beta-M C D$.

3) Theoretical results and assignment

The calculated structures are shown in Figure 6. It should be noticed here that we do not show all the calculated structures but only the most stable member of structural groups with similar structural hydrogen-bonding topology.

a) $\beta-M C D$ complex with L-TyrH ${ }^{+}$

Figures $5 b-e$ shows the most relevant calculated IR spectra of the $\beta-M C D$ complex with L-TyrH ${ }^{+}$. The most stable L-TyrH ${ }^{+}-\beta-M C D$ structure, $\mathrm{L}-\mathrm{TyrH}^{+}-\mathrm{fbb}_{\mathrm{d}}$, has a free $\mathrm{OH}_{\text {phenol, }}$ a bound $\mathrm{OH}_{\text {acid }}$ to $\mathrm{O}_{\alpha}$, and bound $\mathrm{NHs}$ to $\mathrm{O}_{\text {prim }}$ and $\mathrm{O}_{\text {sec. }}$. Another L-TyrH ${ }^{+}$-fbb $b_{d}$ structure was found, which differs from the most stable $\mathrm{fbb}_{\mathbf{d}}$ by the fact that $\mathrm{OH}_{\text {acid }}$ is bound to $\mathrm{O}_{\text {prim }}$ instead of $\mathrm{O}_{\alpha}$ and the aromatic part of the molecule is located outside the cavity. We hereafter denote the former and the latter $\mathbf{f b b}_{\mathrm{d}}$ conformers as L-TyrH $\mathrm{H}^{+}$ $f b_{o \alpha} b_{d}$ and L-TyrH ${ }^{+}-f_{o p r i m} b_{d}$, respectively. In L-TyrH $H^{+}-\mathbf{f b b}$, the complex is bound by an interaction between $\mathrm{OH}_{\text {acid }}$ and $\mathrm{O}_{\text {prim }}$ and two modest interactions between $\mathrm{NHs}$ and $\mathrm{O}_{\alpha}$. Besides the free- $\mathrm{OH}_{\text {phenol }}$ conformational families ("amino-bound"), a "phenol-bound" conformer, L-TyrH+-bbf, was found, in which $\mathrm{OH}_{\text {phenol }}$ and $\mathrm{OH}_{\text {acid }}$ are bound to $\mathrm{O}_{\alpha}$ and $\mathrm{O}_{\text {sec }}$, respectively, while the $\mathrm{NHs}$ are free. However, we

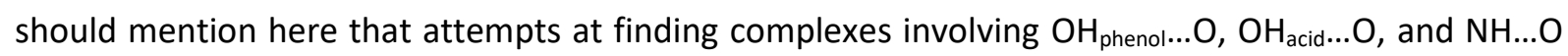
simultaneously (bbb-type structure) have failed.

The experimental spectrum is well reproduced by the superposition of the calculated IR spectra of the three amino-bound conformers or their conformational variants. The band at $3635 \mathrm{~cm}^{-1}$ is ascribed to the free $v\left(\mathrm{OH}_{\text {phenol }}\right)$ of the amino-bound conformers with a slight difference between observed and calculated frequencies. This may arise from the limited calculation level (cam-B3LYP/6-31G(d, p)) due to the size of the system. The doublet in the $3350-3430 \mathrm{~cm}^{-1}$ range is assigned to the superposition of free $v(\mathrm{NH})$ of the amino-bound conformers. Although the calculated frequency of bound $v\left(\mathrm{OH}_{\text {phenol }}\right)$ of bbf is expected in the same range, its contribution to the band at $\sim 3400 \mathrm{~cm}^{-1}$ should be minor. This is because the calculated IR intensity of $v\left(\mathrm{OH}_{\text {phenol }}\right)$ of bbf is much larger than that of $v(\mathrm{NH})$ of the aminobound conformers. Considering that the bound phenolic $\mathrm{v}(\mathrm{OH})$ of the phenol- $\mathrm{NH}_{3}$ complex remains sharp even when the interaction is strong enough to shift its frequency down to $3294 \mathrm{~cm}^{-1}$, 41 the band 
at $\sim 3400 \mathrm{~cm}^{-1}$ would be more prominent if the contribution of bbf were sizable. Last, the broad absorption in the $3100-3200 \mathrm{~cm}^{-1}$ range is characteristic of the strongly bound $v(\mathrm{NH})$ that exists in the three complexes.

b) $\beta$-MCD complex with D-TyrH

Figure $5 \mathrm{~g}$-e shows the calculated IR spectra of the $\beta$-MCD complex with D-TyrH ${ }^{+}$. The most stable conformer was found in D-TyrH ${ }^{+}-\beta-M C D$, which is consistent with the larger intensity of the peak due to the complex with D-TyrH ${ }^{+}$over L-TyrH ${ }^{+}$in the mass spectrum shown in Figure 2. The most stable conformer, $\mathrm{D}-\mathrm{TyrH}^{+}-\mathrm{fbb}_{\mathrm{d}}$, is bound by $\mathrm{OH}_{\text {acid }} . . \mathrm{O}_{\text {sec }}, \mathrm{NH}_{\text {... }} \mathrm{O}_{\text {sec }}$, and $\mathrm{NH} . . . \mathrm{O}_{\text {prim }}$ interactions. D- $\mathrm{TyrH}^{+}-\mathrm{fbb}$ is bound by an interaction between $\mathrm{OH}_{\text {acid }}$ and $\mathrm{O}_{\alpha}$ and an interaction between $\mathrm{NH}$ and $\mathrm{O}_{\text {sec. }}$. Two structures were calculated for phenol-bound D-TyrH ${ }^{+}-\beta-M C D$, namely, D-TyrH ${ }^{+}-\mathbf{b f b}_{\mathbf{d}}$ and D-TyrH $\mathrm{H}^{+}-\mathbf{b} \mathbf{b b}$. We will discard $\mathbf{b f b}_{\mathbf{d}}$ in what follows because its free $\mathrm{OH}_{\text {acid }}$ is not compatible with the experimental results. In bbb, two $\mathrm{O}_{\text {sec }}$ atoms interact with $\mathrm{NH}$ and $\mathrm{OH}_{\text {acid }}$ while $\mathrm{OH}_{\text {phenol }}$ interacts with the $\alpha$-glycosidic linkage. The aromatic part of the molecule is fully inserted in the cavity.

The experimental IRPD spectrum is not well reproduced by the superposition of the calculated IR spectra of the amino-bound structures only and requires inclusion of phenol-bound complexes. In particular, the band at $3432 \mathrm{~cm}^{-1}$ is assigned to the bound $v\left(\mathrm{OH}_{\text {phenol }}\right)$ of the bbb structure due to the close agreement in wavenumber. Another characteristic band at $3100 \mathrm{~cm}^{-1}$ is consistent with the strongly bound $v(\mathrm{NH})$ of $\mathbf{b b b}$. This suggests that $\mathbf{b b b}$, which is less stable in the gas phase, is more stable in methanol solution and its conformation is kinetically trapped during the ESI process, as observed in the isoleucineH ${ }^{+}$- or alanineH ${ }^{+}-\beta-\mathrm{MCD}$ complex. ${ }^{18}{ }^{19}$ This indicates that the amino-bound and phenol-bound D-TyrH $-\beta-M C D$ complexes coexist in our experimental conditions. However, the limitations of the harmonic approximation and the difficulty of calculating reliable intensities and bandwidth for hydrogen-bonded systems, does not allow determining the very precise relative abundance of the two types of structure.

c) Origin of the chiral discrimination.

Both ESI TOF mass spectra obtained under cryogenic ion trap conditions and Q-MS spectra at room temperature show a larger intensity of the peak assigned to the D-Tyr complex. This result contradicts the conclusions from previous guest-exchange reactions experiments in the gas phase, which indicate that the $\mathrm{D}$ enantiomer of $\mathrm{TyrH}^{+}$is easier to displace by an amine, with a ratio of the displacement rate constants $k_{\mathrm{L}} / k_{\mathrm{D}}$ of $0.66 .{ }^{5}$ Several factors can explain this apparent contradiction. First, the geometry proposed by Ramirez et al. for the L-TyrH ${ }^{+}-\beta-M C D$ and D-TyrH $-\beta-M C D$ complexes both involve $\mathrm{NH}$ bonding and free phenol $\mathrm{OH}$. Although $\mathrm{NH}$-bound complexes are also the most stable forms calculated here, our spectroscopic results clearly point at the coexistence of $\mathrm{OH}$ and $\mathrm{NH}$ bound species for the 
D-TyrH $-\beta-M C D$ complex. This could be due to differences in the ESI conditions used for the exchange reactions (50-50 water methanol mixture) and those used for the experiments reported here (pure methanol solutions). The second possible explanation is that the enantioselectivity quantified by Lebrilla and co-workers rests on the displacement of the studied amino acid by an amine. This reaction probably encompasses different steps; not only a simple displacement reaction, but also a net proton transfer to the amine with the leaving amino acid being neutral. It is possible that the observed enantioselectivity depends on one of these steps, in particular on energy barriers, and not only on the stability of the complexes.

The chiral discrimination observed here probably arises from two facts: (1) enantiomerically different molecular structures in amino-bound conformations and (2) presence (absence) of phenol-bound conformations. Figure S4 shows the mass spectrum of a solution containing MCD, D-phenylalanine (Phe), and isotope $\left({ }^{13} \mathrm{C},{ }^{15} \mathrm{~N}\right)$ labelled L-Phe. The observed mass spectrum indicates that $\mathrm{PheH}^{+}$, in which the phenolic $\mathrm{OH}$ is substituted by hydrogen, has little enantioselectivity to MCD. This supports that chiral discrimination arises from $\mathrm{H}$-bonding of phenolic $\mathrm{OH}$ to MCD. In D-TyrH ${ }^{+}-\mathrm{fb}_{\mathbf{o \alpha}_{\alpha}} \mathbf{b}_{\mathrm{d}}$, the structure of $\mathrm{TyrH}^{+}$is more folded and its aromatic ring is more tilted than in L-TyrH ${ }^{+}-\mathrm{fbb}_{\mathrm{d}}$. In D-TyrH ${ }^{+}$-fbb also, $\mathrm{TyrH}^{+}$ is more folded and more deeply included in the cavity than what is observed in L-TyrH $\mathrm{H}^{+}-\mathbf{f b b}$. The folded geometry in $\mathrm{D}-\mathrm{TyrH}^{+}-\beta-\mathrm{MCD}$ is favourable because the hydrophobic aromatic moiety is less exposed to hydrophilic solvent (methanol) molecules. Moreover, the D-Tyr complex is able to coordinate NH, $\mathrm{OH}_{\text {phenol, }}$ and $\mathrm{OH}_{\text {acid }}$ simultaneously (bbb-type structure), whereas L-Tyr is not able to do so. The D$\mathrm{TyrH}^{+}$-bbb complex shows full insertion of the hydrophobic benzene ring within the cavity. In addition, it is possible that the bound phenol moiety imposes an increased stiffness in D-TyrH ${ }^{+}$-bbb resulting to higher dissociation barriers, hence a larger abundance of the D- $\mathrm{yyrH}^{+}$complexes. Close inspection of the binding sites in D-TyrH ${ }^{+}$-bbb gives a clue to understand why L-TyrH $\mathrm{H}^{+}$-bbb is not favoured. In D$\mathrm{TyrH}^{+}$-bbb, $\mathrm{OH}_{\text {phenol }}$ is bonded to an $\mathrm{O}_{\alpha}$ atom. $\mathrm{OH}_{\text {acid }}$ and $\mathrm{NH}$ are bonded to the $\mathrm{O}_{\text {sec }}$ atoms in the fourth and sixth $\mathrm{CH}_{3} \mathrm{O}_{\text {sec }}$ moiety from the $\mathrm{O}_{\alpha}$ atom in a clockwise way viewed above the secondary rim (Figure 7). The $\mathrm{CH}_{3} \mathrm{O}_{\text {sec }}$ moieties are alternatively pointed inward and outward, forming a helical structure in the secondary rim surface. In L-TyrH $-\beta-M C D, \mathrm{OH}_{\text {acid }}$ and $\mathrm{NH}$ should be bonded to the $\mathrm{O}_{\text {sec }}$ atoms in the fourth and sixth $\mathrm{CH}_{3} \mathrm{O}_{\text {sec }}$ moiety, respectively, in a counterclockwise way assuming the identical binding pattern. However, because these $\mathrm{CH}_{3} \mathrm{O}_{\text {sec }}$ moieties are pointed outward, $\mathrm{L}-\mathrm{TyrH}^{+}-\beta-\mathrm{MCD}$ allows neither $\mathrm{OH}_{\text {acid }}$ nor $\mathrm{NH}$ to be bonded to the $\mathrm{O}_{\text {sec. }}$. Of course, the conformational flexibility of $\mathrm{TyrH}^{+}$and multiple binding patterns should be taken into account for detail discussions, however, the simple model suggests that the helical structure of $\beta-M C D$ with no mirror symmetry orthogonal to the secondary rim surface contributes to enantiomerically variable binding motifs. 


\section{Conclusion}

ESI mass spectrum of the protonated complexes of $\beta-\mathrm{MCD}$ with the two enantiomers of $\mathrm{TyrH}^{+}$was measured and revealed that D-TyrH ${ }^{+}$complex is more abundant than that of L-TyrH ${ }^{+}$. IR spectra of these complexes were measured using the cryogenic ion trap technique. These complexes are formed from neutral $\beta-\mathrm{MCD}$ and the protonated amino acid, and in all the structures observed, the $\mathrm{COOH}$ is in strong interaction with the cavity. This contrasts to the case of the $\mathrm{TyrH}^{+} /$crown ether complexes. ${ }^{42}$ Structures involving interaction between $\beta-M C D$ and both $\mathrm{COOH}$ and $\mathrm{NH}_{3}{ }^{+}$are observed for both enantiomers. However, D-TyrH ${ }^{+}$complex shows more folded geometry, which is favourable because the hydrophobic aromatic moiety is less exposed to hydrophilic solvent molecules. The structure involving the interaction between $\beta-\mathrm{MCD}$ and the phenolic $\mathrm{OH}$, namely, the D-TyrH ${ }^{+}-\mathbf{b b b}_{\text {complex, }}$ is observed only for D-TyrH ${ }^{+}$. Despite being higher in energy in the gas phase, this structure is kinetically trapped during the ESI process and shows three interaction points between the amino acid and the cavity, in which it is fully inserted. This result contrasts with the case of D- and L-isoleucine or D- and L-alanine in which only surface binding is observed and points towards the importance of the $\mathrm{OH}$ group of tyrosine. ${ }^{18,19}$ Interestingly, the $\beta$-MCD cavity is large enough so that the recognition process does not occur through size effects. Instead, specific interactions are responsible for the recognition process. These experiments bring molecular-scale understanding on how the cyclodextrin cavity recognises the chirality of a host molecule. Further work on non-aromatic amino acids and other sugarbuilt cavity is in progress.

\section{Acknowledgement}

A.Z. acknowledges travel support from the World Research Hub Initiative (WRHI) of Tokyo Institute of Technology. We acknowledge the use of the computing facility cluster MésoLUM of the LUMAT federation (FR LUMAT 2764). This work was supported in part by KAKENHI (JP205104008) on innovative area (2503), KAKENHI (JP19H05527, JP18H01938, JP19K23624) of JSPS, World Research Hub Initiatives in Tokyo Institute of Technology, the Cooperative Research Program of the "Network Joint Research Center for Materials and Devices" from the Ministry of Education, Culture, Sports, Science and Technology (MEXT), Japan, and the RIKEN Pioneering Project, "Fundamental Principles Underlying the Hierarchy of Matter: A Comprehensive Experimental Study". The computations were in part performed at the Research Center for Computational Science, Okazaki, Japan.

\section{Author contribution}


A.Z., M.F. and S.I. conceived and designed the project. K.H. and S.I. conducted the experiments. Y.M., K.H. and S.I. measured and analysed the spectra. K.H., Y.M., and A.Z. performed and analysed the quantum chemical calculations. A.Z., K.H., M.F. and S.I. co-wrote the paper.

\section{References}

1. X. Li, M. Porcino, C. Martineau-Corcos, T. Guo, T. Xiong, W. Zhu, G. Patriarche, C. Pechoux, B. Perronne, A. Hassan, R. Kummerle, A. Michelet, A. Zehnacker-Rentien, J. Zhang and R. Gref, International Journal of Pharmaceutics, 2020, 585.

2. R. Mejia-Ariza, L. Grana-Suarez, W. Verboom and J. Huskens, Journal of Materials Chemistry B, 2017, 5, 36-52.

3. Y. Xiao, S. C. Ng, T. T. Y. Tan and Y. Wang, Journal of Chromatography A, 2012, 1269, 52-68.

4. D. W. Armstrong and W. Demond, Journal of Chromatographic Science, 1984, 22, 411-415.

5. S. Ahn, J. Ramirez, G. Grigorean and C. B. Lebrilla, Journal of the American Society for Mass Spectrometry, 2001, 12, 278-287.

6. M. Lammerhofer, Journal of Chromatography A, 2010, 1217, 814-856.

7. S. Li and W. C. Purdy, Chemical Reviews, 1992, 92, 1457-1470.

8. G. Grigorean, J. Ramirez, S. H. Ahn and C. B. Lebrilla, Analytical Chemistry, 2000, 72, 42754281.

9. C. B. Lebrilla, Accounts of Chemical Research, 2001, 34, 653-661.

10. W. A. Tao, F. C. Gozzo and R. G. Cooks, Analytical Chemistry, 2001, 73, 1692-1698.

11. A. Zehnacker, International Reviews in Physical Chemistry, 2014, 33, 151-207.

12. A. Sen, K. Le Barbu-Debus, D. Scuderi and A. Zehnacker-Rentien, Chirality, 2013, 25, 436-443.

13. D. Scuderi, P. Maitre, F. Rondino, K. Le Barbu-Debus, V. Lepere and A. Zehnacker-Rentien, Journal of Physical Chemistry A, 2010, 114, 3306-3312.

14. M. E. Crestoni, B. Chiavarino, D. Scuderi, A. Di Marzio and S. Fornarini, Journal of Physical Chemistry B, 2012, 116, 8771-8779.

15. B. Botta, C. Fraschetti, F. R. Novara, A. Tafi, F. Sacco, L. Mannina, A. P. Sobolev, J. Mattay, M. C. Letzel and M. Speranza, Organic \& Biomolecular Chemistry, 2009, 7, 1798-1806.

16. A. Filippi, C. Fraschetti, L. Guarcini, C. Zazza, T. Ema and M. Speranza, Chemphyschem, 2017, 18, 2475-2481.

17. A. Filippi, C. Fraschetti, S. Piccirillo, F. Rondino, B. Botta, I. D'Acquarica, A. Calcaterra and M. Speranza, Chemistry-a European Journal, 2012, 18, 8320-8328.

18. S. S. Lee, J. U. Lee, J. H. Oh, S. Park, Y. Hong, B. K. Min, H. H. L. Lee, H. I. Kim, X. L. Kong, S. Lee and H. B. Oh, Physical Chemistry Chemical Physics, 2018, 20, 30428-30436.

19. S. S. Lee, S. Park, Y. Hong, J. U. Lee, J. H. Kim, D. Yoon, X. Kong, S. Lee and H. Bin Oh, Physical Chemistry Chemical Physics, 2017, 19, 14729-14737.

20. T. R. Rizzo and O. V. Boyarkin, eds., Gas-Phase IR Spectroscopy and Structure of Biological Molecules, Springer-Verlag Berlin, Berlin, 2015.

21. J. Klyne, A. Bouchet, S. Ishiuchi, M. Fujii, M. Schneider, C. Baldauf and O. Dopfer, Physical Chemistry Chemical Physics, 2018, 20, 28452-28464.

22. M. Tamura, T. Sekiguchi, S.-I. Ishiuchi, A. Zehnacker-Rentien and M. Fujii, The journal of physical chemistry letters, 2019, DOI: 10.1021/acs.jpclett.9b00184.

23. S. Ishiuchi, H. Wako, D. Kato and M. Fujii, Journal of Molecular Spectroscopy, 2017, 332, 4551. 
24. S. Ishiuchi, Y. Sasaki, J. M. Lisy and M. Fujii, Physical Chemistry Chemical Physics, 2019, 21, 561-571.

25. D. A. Wild, Z. M. Loh, R. L. Wilson and E. J. Bieske, Chem. Phys. Lett., 2003, 369, 684-690.

26. M. Z. Kamrath, E. Garand, P. A. Jordan, C. M. Leavitt, A. B. Wolk, M. J. Van Stipdonk, S. J. Miller and M. A. Johnson, Journal of the American Chemical Society, 2011, 133, 6440-6448.

27. M. J. Frisch, G. W. Trucks, H. B. Schlegel, G. E. Scuseria, M. A. Robb, J. R. Cheeseman, G. Scalmani, V. Barone, G. A. Petersson, H. Nakatsuji, X. Li, M. Caricato, A. V. Marenich, J. Bloino, B. G. Janesko, R. Gomperts, B. Mennucci, H. P. Hratchian, J. V. Ortiz, A. F. Izmaylov, J. L. Sonnenberg, Williams, F. Ding, F. Lipparini, F. Egidi, J. Goings, B. Peng, A. Petrone, T. Henderson, D. Ranasinghe, V. G. Zakrzewski, J. Gao, N. Rega, G. Zheng, W. Liang, M. Hada, M. Ehara, K. Toyota, R. Fukuda, J. Hasegawa, M. Ishida, T. Nakajima, Y. Honda, O. Kitao, H. Nakai, T. Vreven, K. Throssell, J. A. Montgomery Jr., J. E. Peralta, F. Ogliaro, M. J. Bearpark, J. J. Heyd, E. N. Brothers, K. N. Kudin, V. N. Staroverov, T. A. Keith, R. Kobayashi, J. Normand, K. Raghavachari, A. P. Rendell, J. C. Burant, S. S. Iyengar, J. Tomasi, M. Cossi, J. M. Millam, M. Klene, C. Adamo, R. Cammi, J. W. Ochterski, R. L. Martin, K. Morokuma, O. Farkas, J. B. Foresman and D. J. Fox, Journal, 2016.

28. http://cccbdb.nist.gov/vibscalejust.asp).

29. K. A. Kellersberger, C. Dejsupa, Y. J. Liang, R. M. Pope and D. V. Dearden, International Journal of Mass Spectrometry, 1999, 193, 181-195.

30. MacroModel version 9.8; ed. Schrödinger, LLC: New York, NY, 2010.

31. E. Harder, W. Damm, J. Maple, C. Wu, M. Reboul, J. Y. Xiang, L. Wang, D. Lupyan, M. K. Dahlgren, J. L. Knight, J. W. Kaus, D. S. Cerutti, G. Krilov, W. L. Jorgensen, R. Abel and R. A. Friesner, Journal of Chemical Theory and Computation, 2016, 12, 281-296.

32. J. Tomasi, B. Mennucci and R. Cammi, Chemical Reviews, 2005, 105, 2999-3093.

33. J. A. Stearns, S. Mercier, C. Seaiby, M. Guidi, O. V. Boyarkin and T. R. Rizzo, Journal of the American Chemical Society, 2007, 129, 11814-11820.

34. R. K. Sinha, B. Chiavarino, M. E. Crestoni, D. Scuderi and S. Fornarini, International Journal of Mass Spectrometry, 2011, 308, 209-216.

35. Y. Inokuchi, T. Haino, R. Sekiya, F. Morishima, C. Dedonder, G. Feraud, C. Jouvet and T. Ebata, Physical Chemistry Chemical Physics, 2015, 17, 25925-25934.

36. A. Fujihara, T. Sato and S. Hayakawa, Chem. Phys. Lett., 2014, 610, 228-233.

37. F. Ben Nasr, I. Alata, D. Scuderi, V. Lepere, V. Brenner, N.-E. Jaidane and A. Zehnacker, Physical Chemistry Chemical Physics, 2019, 21, 15439-15451.

38. Y. Inokuchi, R. Kusaka, T. Ebata, O. V. Boyarkin and T. R. Rizzo, Chemphyschem, 2013, 14, 649660.

39. A. M. Rijs, G. Ohanessian, J. Oomens, G. Meijer, G. von Helden and I. Compagnon, Angewandte Chemie-International Edition, 2010, 49, 2332-2335.

40. R. H. Wu and T. B. McMahon, Chemphyschem, 2008, 9, 2826-2835.

41. A. Iwasaki, A. Fujii, T. Watanabe, T. Ebata and N. Mikami, Journal of Chemical Physics, 1996, 100, 16053-16057.

42. C. N. Stedwell, J. F. Galindo, K. Gulyuz, A. E. Roitberg and N. C. Polfer, Journal of Physical Chemistry A, 2013, 117, 1181-1188. 

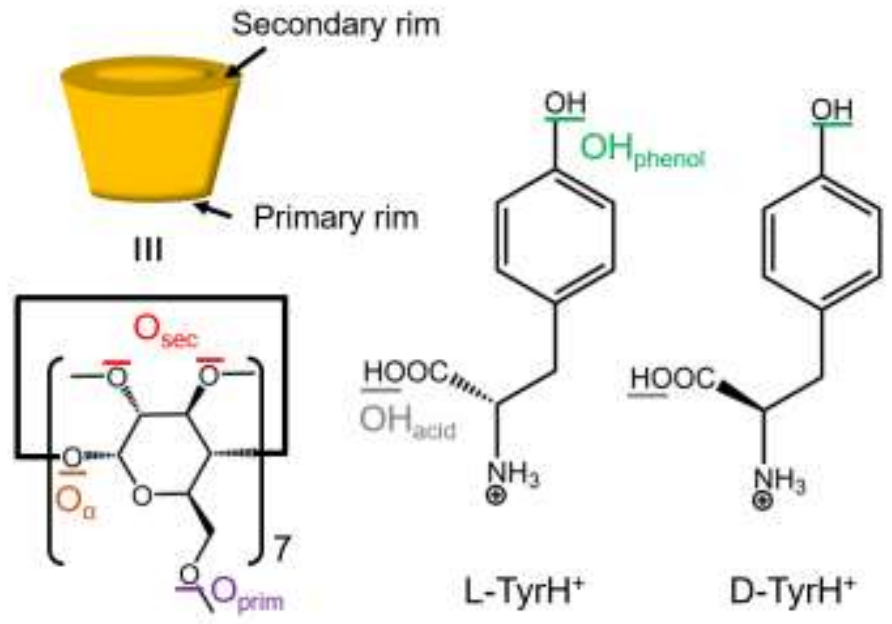

$\mathrm{L}-\mathrm{TyrH}^{+} \quad$ D-TyrH $\mathrm{H}^{+}$

\section{$\beta-M C D$}

Figure 1 Host and guest molecules used in this study. $\mathrm{O}_{\alpha}, \mathrm{O}_{\text {prim, }}$ and $\mathrm{O}_{\text {sec }}$ correspond to the oxygen atoms of 1,4- $\alpha$-glyosidic linkage, primary rim group, and secondary rim group, respectively. $\mathrm{OH}_{\text {phenol }}$ and $\mathrm{OH}_{\text {acid }}$ correspond to phenolic and carboxylic $\mathrm{OH}$, respectively.

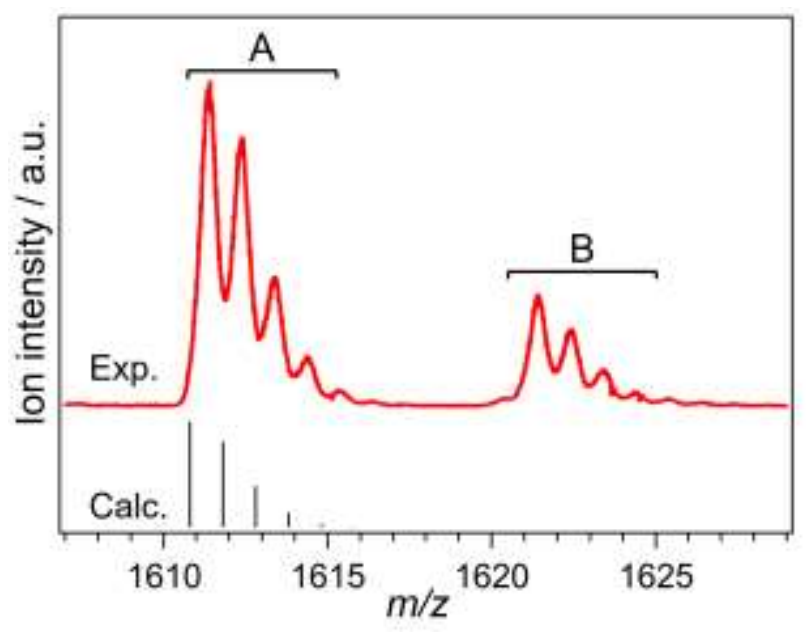

Figure $2 \mathrm{ESI}$ mass spectrum of the solution containing $\beta-\mathrm{MCD}$, D-Tyr, and isotope-labelled L-Tyr, in the region of the mass of the complex with the calculated isotope pattern of D-TyrH ${ }^{+}-\beta-M C D$. Peaks $\mathrm{A}$ and $\mathrm{B}$ correspond to the complex with $\mathrm{D}-\mathrm{TyrH}^{+}$and ${ }^{15} \mathrm{~N},{ }^{13} \mathrm{C}$-substituted $\mathrm{L}-\mathrm{TyrH}^{+}$, respectively. The concentrations of $D$ - and L-Tyr are equivalent. 


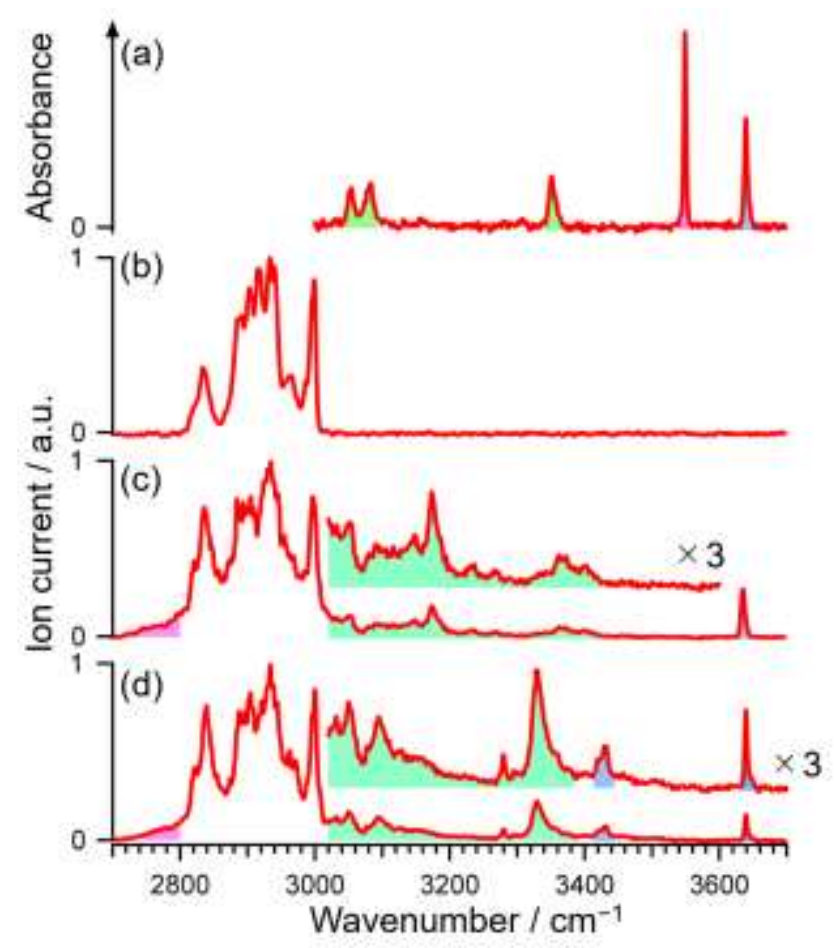

Figure 3 (a) IR-UV ion dip spectrum of conformer A of L-TyrH ${ }^{+}$(Nomenclature of Reference 32) and IRPD spectra of $\mathrm{H}_{2}$-tagged (b) protonated $\beta-M C D$, (c) L-TyrH ${ }^{+}-\beta-M C D$, and (d) D-TyrH ${ }^{+}-\beta-M C D$ in the $3 \mu \mathrm{m}$ region. Blue-, purple-, and green-coloured areas correspond to the bands assigned to $v\left(\mathrm{OH}_{\text {phenol }}\right), v\left(\mathrm{OH}_{\text {acid }}\right)$, and $v(\mathrm{NH})$, respectively.

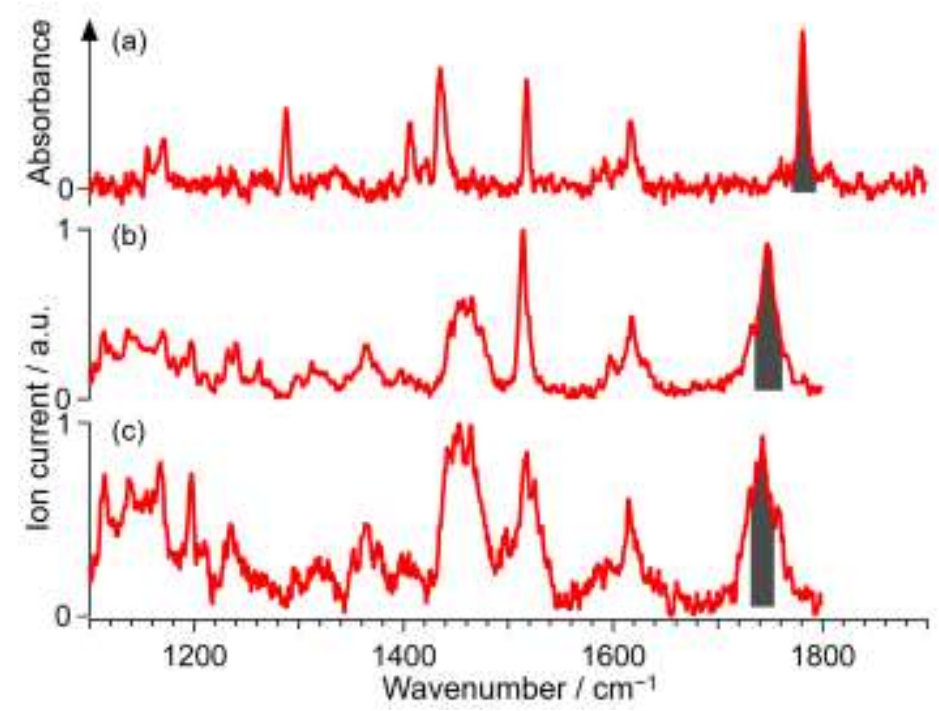

Figure 4 (a) IR-UV ion dip spectrum of conformer A of L-TyrH ${ }^{+}$(Nomenclature of Reference 33) and IRPD spectra of $\mathrm{H}_{2}$-tagged (b) $\mathrm{L}$ - and (c) $D-\mathrm{TyrH}^{+}-\beta-\mathrm{MCD}$ in the fingerprint region. The grey area corresponds to the bands assigned to $v(\mathrm{CO})$. 

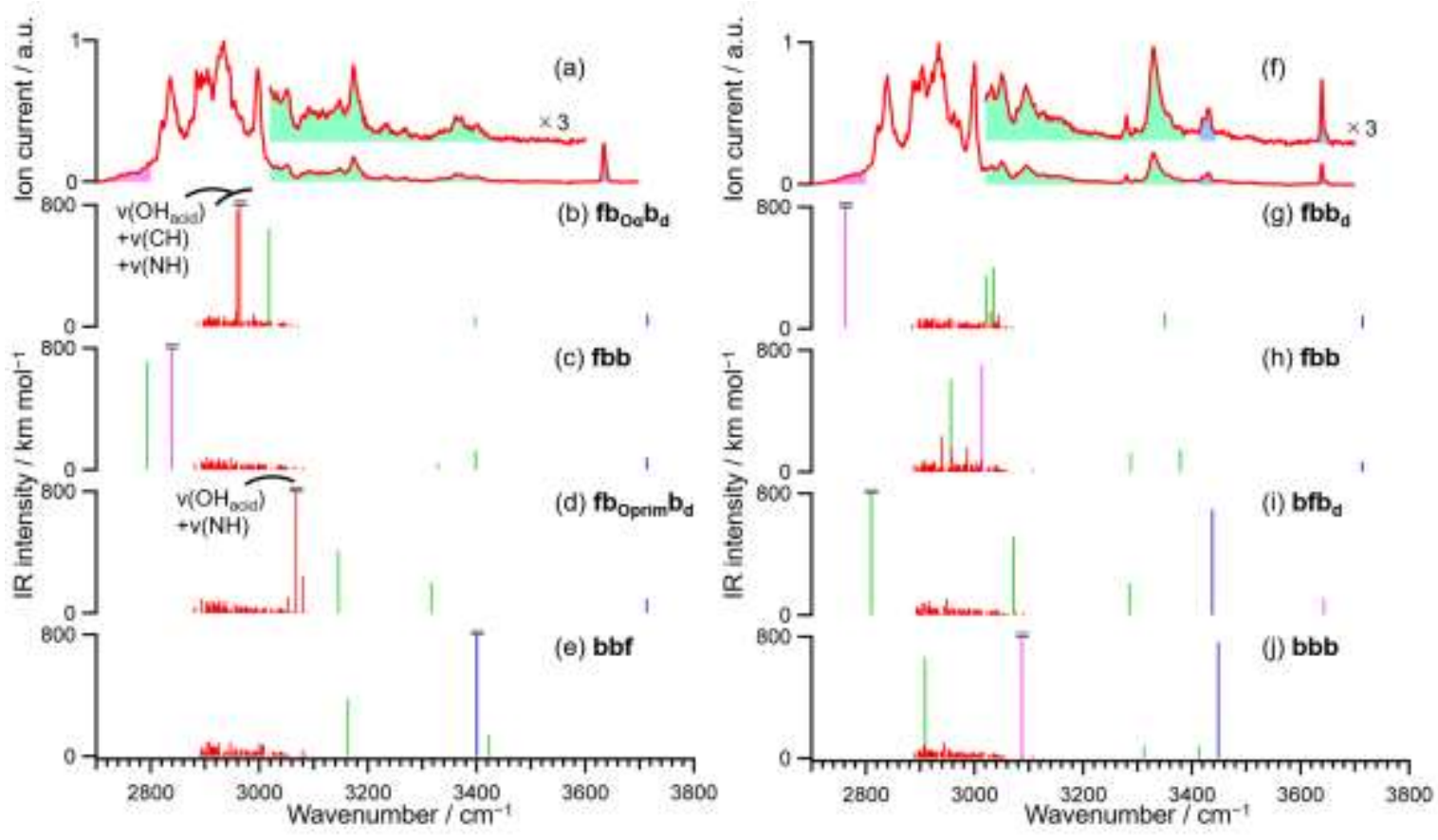

Figure 5 Experimental IRPD spectra of (a) $L$ - and (f) $D-\mathrm{TyrH}^{+}-\beta-\mathrm{MCD}$ and theoretical IR spectra of (b-e) L- and (g-j) D-TyrH ${ }^{+}-\beta-M C D$. The theoretical IR spectra were obtained at the cam-B3LYP/6$31 \mathrm{G}(\mathrm{d}, \mathrm{p})$ level with a scaling factor of 0.961 . Blue-, purple-, and green-colored lines correspond to the bands assigned to $v\left(\mathrm{OH}_{\text {phenol }}\right), v\left(\mathrm{OH}_{\text {acid }}\right)$, and $v(\mathrm{NH})$, respectively.

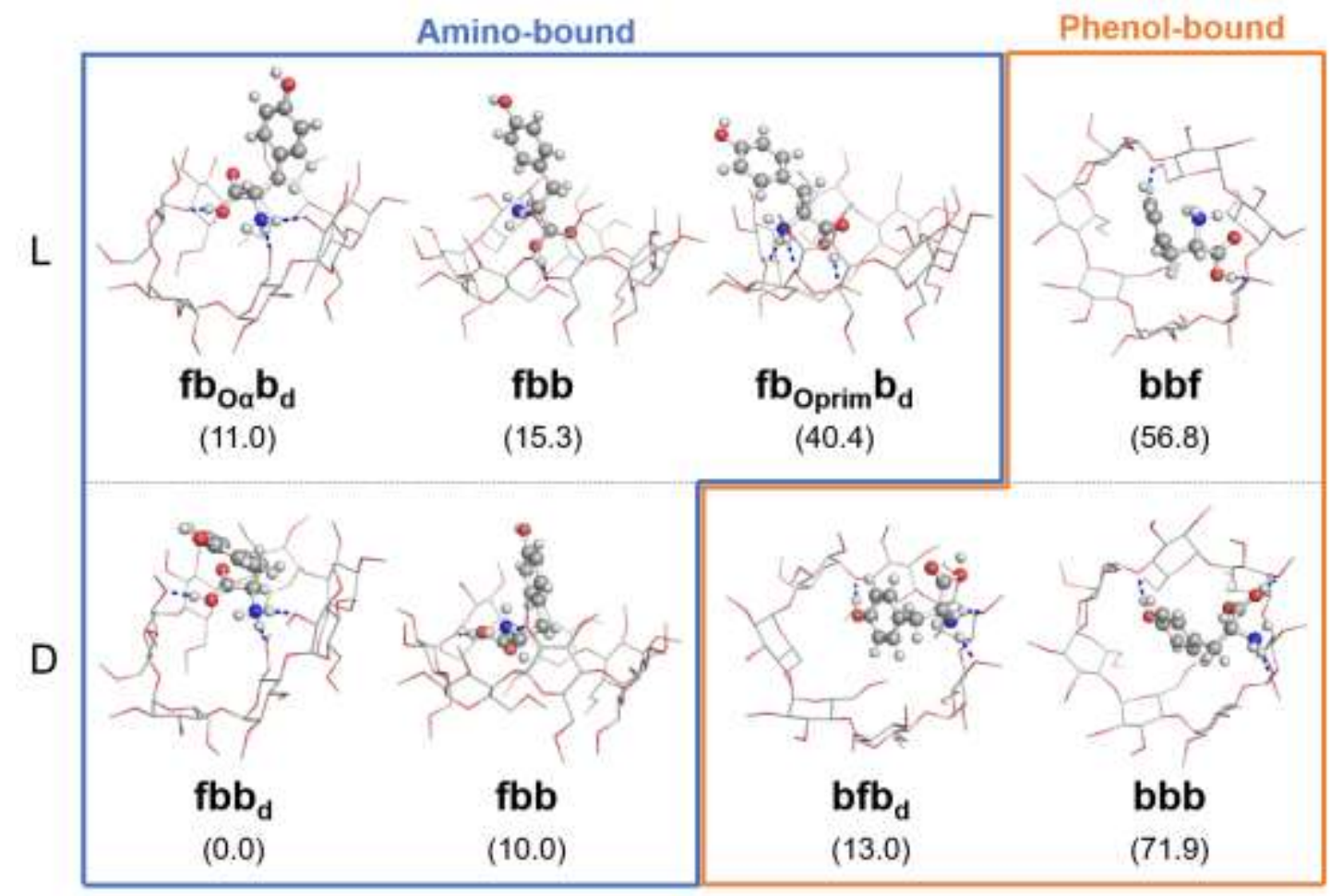

Figure 6 Optimized structures of L-/D-TyrH ${ }^{+}-\beta-M C D$. The number in parenthesis is the relative Gibbs free energy (in $\mathrm{kJ} \mathrm{mol}^{-1}$ ) compared to the most stable complex D-TyrH ${ }^{+}-\mathrm{fbb}_{\mathrm{d}}$. 


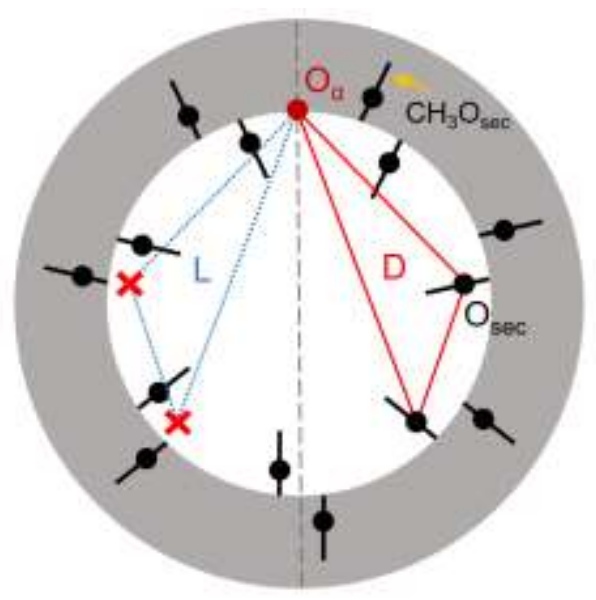

Figure 7 Schematic illustration of the interaction points in the secondary rim surface (grey shaded area) of $\mathrm{D}-\mathrm{TyrH}^{+}$-bbb (red solid triangle) and the corresponding L-TyrH $\mathrm{H}^{+} \beta-\mathrm{MCD}$ structure (blue dotted triangle) assuming an identical binding pattern. Black line represents $\mathrm{CH}_{3} \mathrm{O}_{\text {sec }}$ moiety. The red and black dots correspond to $\mathrm{O}_{\alpha}$ and $\mathrm{O}_{\text {sec }}$, respectively. 


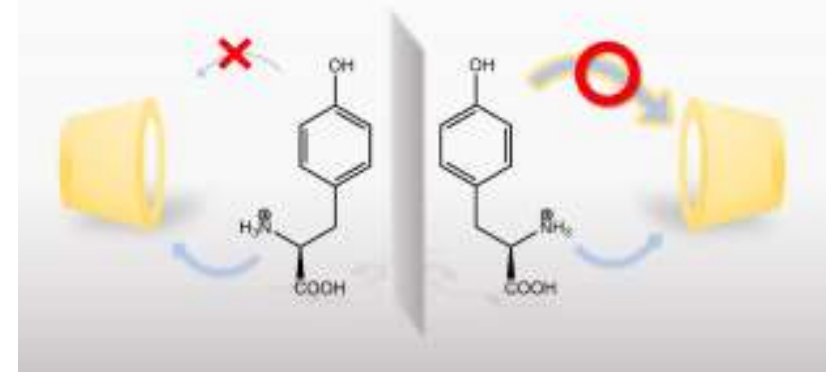

Different binding motifs between the two enantiomers of protonated tyrosine $\left(\mathrm{TyrH}^{+}\right)$and permethylated $\beta$-cyclodextrin ( $\beta-M C D)$ lead to chiral discrimination. Cryogenic ion trap infrared spectroscopy reveals that while the structure of the complex of L-TyrH ${ }^{+}$with $\beta-\mathrm{MCD}$ involve "amino-bound" conformational family, both "amino-bound" and "phenol-bound" coexist in the DTyrH ${ }^{+}-\beta-M C D$ complex. 